\title{
The Relationship between Assessment Skills and Nursing Knowledge
}

\author{
Keiko Aizu1*, Kasumi Mikami1, Ryoko Tsuchiya², Mayumi Shimizu², Nanae Kojima³, \\ Naoki Oka4, Yoshiko Nishizawa ${ }^{1}$ \\ ${ }^{1}$ School of Health Sciences, Hirosaki University, Aomori, Japan \\ ${ }^{2}$ School of Medicine \& Hospital, Hirosaki University, Aomori, Japan \\ ${ }^{3}$ Health Service Center, Hiroshima University, Hiroshima, Japan \\ ${ }^{4}$ School of Education, Hiroshima University, Hiroshima, Japan \\ Email: *aizu@hirosaki-u.ac.jp
}

Received 5 November 2015; accepted 18 December 2015; published 21 December 2015

Copyright (C) 2015 by authors and Scientific Research Publishing Inc.

This work is licensed under the Creative Commons Attribution International License (CC BY).

http://creativecommons.org/licenses/by/4.0/

(c) (i) Open Access

\section{Abstract}

This research aimed to clarify the relationship between nursing students' levels of structural knowledge and assessment skills. Participants were 17 third-year university students majoring in nursing who participated individually in the experiments. The experiments included a nursingscene task, free-recall task, and sorting task. Experiments were conducted before and after their clinical practice. Each student's level of structural knowledge was calculated from the free-recall and sorting task responses, and each student's assessment skill was calculated from the nursingscene task responses. Levels of structural knowledge were significantly higher in post-examination compared with pre-examination $(p<0.01)$. Assessment skills scores were also significantly higher in post-examination compared with pre-examination $(p<0.001)$. When comparing assessment skills in relation to levels of structural knowledge, the high-structured group $(n=5)$ had significantly higher scores for their conclusions and reasons than the low-structured group $(n=5)(p<$ 0.001). Information-collecting scores in the task case did not differ between the low- and highstructured groups, although the high-structured group tended to mention information that was not included in the task case $(p<0.10)$. Well-structured knowledge of students in the high-structured group seemed to enable them to acquire and activate highly related information during assessment. Students in the high-structured group apparently made assessments not only by using information given but also by over viewing information comprehensively.

\section{Keywords}

Structural Knowledge, Assessment, Clinical Practice, Cognitive Psychology

\footnotetext{
*Corresponding author.
} 


\section{Introduction}

Recently, nurses are required to have a high level of clinical judgment due to changes in the types of diseases of aged patients and advances in medical technology. The thinking process that a nurse goes through when caring for patients is called the "nursing process" which includes the processes of "assessment", "nursing diagnosis", "planning", "implementing" and "evaluation" [1]. Specifically, assessment is the process of collecting patient information, analyzing the situation, and understanding the problem or potential problem [2]. Nurses are required to have high-level skills in assessment given that nursing diagnosis, planning, and implementing are based on assessment.

In clinical practice, nursing students are required to care for patients and provide needed nursing care as licensed nurses do even though they are students. Therefore, even students in clinical practice are expected to have high-level assessment skills. However, Sasabe et al. reported that at graduation, students' self-evaluations of their assessment skills were low [3]. After Sasabe et al. improved the students' assessment skill training, evaluations of their information collecting and analytical skills were relatively higher whereas evaluations of practice or physical assessments to solve problems were low [4]. Sasabe et al. also mentioned the need for continuous college education in the thinking process of nursing [4]. Therefore, high-level assessment skill education for nursing students is very important in nursing education.

Yura and Walsh [5] defined the nursing process as a problem-solving activity to clarify patients' problems and needs in order to provide appropriate care. If the nursing process is indeed a problem-solving activity, knowledge of cognitive psychology will provide a new perspective for developing ways to train assessment skills.

For example, Chi et al. [6] demonstrates that one's knowledge structure affects how one solves problems. Moreover, they found that the structural knowledge of an expert in a particular field is more structured than that of a novice. "Structuring knowledge" is the process of uniting and unifying new knowledge with the knowledge that one already has. It is said that in problem-solving, once certain knowledge is activated, other related knowledge is also activated. Therefore, one can use obtained knowledge to solve problems. The structural knowledge of experts allows them to employ their knowledge to solve a problem effectively. Nurses and nursing students can also effectively use their knowledge for assessment if their knowledge about nursing is well structured.

When considering ways to improve student assessment skills, the relationship between students' structural knowledge and assessment skills must be understood because the amount and structure of knowledge affect the ways of problem-solving. However, there is little research on assessment skills in relation to structural knowledge despite the abundant research on nursing-process education. Both in the United States and in Japan, standardized patients or students' self-assessments by the Objective Structured Clinical Examination (OSCE) are used in assessment education (e.g., Hemming and Jennrich [7], Mitsuki et al. [8]). However, standardized patients and the OSCE focus on students' behaviors and ways of thinking. It is difficult to say whether there are enough studies about the knowledge utilized for assessment.

Therefore, this study aims to clarify the relationship between the structural knowledge of nursing students and their assessment skills.

In this study, we organized the structural knowledge of nursing students through a measure of category clustering. Measurements were made by free recall of verbal materials in experiments. Various measures were developed to clarify the development of memory or structural knowledge. Adjusted-ratio-of-clustering-S (ARCS; Inoki [9]) is a category clustering measurement adopted in this research for its ability to measure clusters even when free recall numbers are small. ARCS is considered suitable for measuring novices' structural knowledge. We evaluated assessment skills through an assessment process using the contents "collecting information" and "nursing problems and causes". A quantified examination of the relationship between structural knowledge and assessment skills becomes possible by evaluating levels of structural knowledge and assessment skills together, which has never been done before. Clarifying differences in assessment skills based on levels of structural knowledge is linked to clarifying the structural knowledge effective in assessment. This would be important for developing an education strategy to improve structural knowledge that is useful for assessment.

\section{Method}

\subsection{Participants}

Participants were 17 third-year university students majoring in nursing. The purpose of this research was ex- 
plained to 35 students who were randomly sampled from the students enrolled in Basic Nursing Practice in the 2014 academic year. Of these students, 17 volunteered to participate in this study.

\subsection{Materials}

Participants were individually interviewed and participated individually in the experiments. Three tasks were administered: a nursing-scene task, free-recall task, and sorting task. One experiment took approximately 60 to $90 \mathrm{~min}$. Experiments were conducted before and after each participant's clinical practice, during the period from July to November 2014.

\subsection{Case Content of the Nursing-Scene Task}

The originally conceived case was about a patient who had increased energy demand five days after lobectomy for lung cancer but who lacked sufficient energy intake (see Table 1 for details).

\subsection{Verbal Materials for the Free-Recall Task}

Fifteen pairs of 30 terms were chosen from a textbook used by the participants. The terms were related to the nutritional-metabolic pattern in Gordon's functional health patterns, which is knowledge needed for assessment. Participants had studied the terms prior to the experiments. Two researchers engaged in nursing education chose the terms so that the terms could be paired with complementary terms. Examples of terms used in the free-recall test are shown in Table 2.

\subsection{Procedures}

\subsubsection{Nursing-Scene Task}

The case was presented on paper to the participants and they were asked to answer orally.

Participants were asked to discuss the problems and factors of the issues in the case. They were asked not only to give conclusions but also to think about how they thought about the case. Participants were asked to assess the problems related to the nutritional-metabolic pattern.

\section{Table 1. Case for the clinical-scene task.}

\section{Instruction:}

Please discuss the problems and factors of the issues in this case. Please discuss how you thought about the case as well as your conclusions. The problems are related to the nutritional-metabolic pattern.

65-year-old man treated with a lobectomy for lung cancer on February 8. His situation on February 13 is given below.

Activity level Although the patient can walk around the ward freely, he is usually found in a supine position on the bed when nurses visit his room. He said "There is nowhere I want to go to".

A meal of gruel (1600 kcal) was served for lunch on February 10 . From February 10 to 13 , he consumed
Eating activity level. After breakfast on February 13 he said "I might not be hungry because I do not walk".

Showering is not allowed yet. Bed-bath is conducted with some assistance. When a nurse hands him a towe he can maintain a sitting position and independently wipes his body. Wiping his back and lower extremities requires assistance.

$\begin{array}{ll} & \text { He can walk to the restroom and self-excrete. } \\ \text { Excretion } & \text { After surgery, there was a little stool on only the } 10^{\text {th }} \text {. }\end{array}$ He said he has no desire to defecate but voiced that "My stomach feels heavy".

$\begin{array}{ccccc} & & \text { February } 12 & \text { Baseline } & 3600-9300 / \mu l \\ \text { WBC } & \text { Admission day } & 5080 \mu / 1 & 4.3-5.54 \mathrm{million} / \mu \mathrm{l} \\ \text { Blood test } & \text { RBC } & 4.7 \mathrm{million} / \mu \mathrm{l} & 4.5 \mathrm{million} / \mu \mathrm{l} & 13.8-16.9 \mathrm{~g} / \mathrm{dl} \\ & \text { Hb } & 14.0 \mathrm{~g} / \mathrm{dl} & 5.8 \mathrm{~g} / \mathrm{dl} & 6.6-8.1 \mathrm{~g} / \mathrm{dl} \\ \text { TP } & 6.7 \mathrm{~g} / \mathrm{dl} & 2.5 \mathrm{~g} / \mathrm{dl} & 4.1-4.9 \mathrm{~g} / \mathrm{dl} \\ & \text { ALB } & 3.5 \mathrm{~g} / \mathrm{dl} & 1.543 \mathrm{mg} / \mathrm{dl} & \text { Under } 0.6 \mathrm{mg} / \mathrm{dl}\end{array}$


Table 2. Verbal materials for the free-recall task.

Meals / Appetite
Exercise / Sleep
Height / Weight
$\mathrm{TP} /$ Alb
Infection / CRP
Intention / Scar
Bed-bath / Foot-bath
Hydration / Dehydration
Bedsores / Wet
Compression / Friction
Assessment / Nursing process
Information gathering /
Communication
Constipation / Bloating
Basal metabolism / Calorie intake
Nutritional condition / Vital signs

\subsubsection{Free-Recall Task}

Experimental procedures were explained to the participants before the task. Terms were visually presented on a personal computer screen for 3 each. To prevent rehearsal using short-term memory, participants were asked to do a calculation task (i.e., adding 10 adjacent numbers for $30 \mathrm{~s}$ ). After the calculation task, participants were asked to recall memorized terms in free order for $120 \mathrm{~s}$. Participants' responses were recorded with an IC recorder. One trial consisted of visual presentation of the terms, the calculation task, and free recall. Six trials were carried out for each participant.

\subsubsection{Sorting Task}

A white board (58 cm width $\times 43 \mathrm{~cm}$ height $)$ and 30 cards $(6 \mathrm{~cm}$ width $\times 1.5 \mathrm{~cm}$ height $)$ each displaying a term were presented to the participants, who were asked to categorize these cards into groups using their own thinking.

\subsection{Analysis}

\subsubsection{Assessment Skills}

We created a checklist to evaluate assessment skills for the nursing-scene task. The checklist's validity was checked by four researchers including two teachers and two clinical experts. The checklist comprised three assessment categories and 34 items: "conclusions and reasons" had 8 items and "information paid attention to" had 26 items, of which 13 items were included in the case and 13 items were not included in the case (Table 3).

\subsubsection{Structural Knowledge}

ARCS scores were used to measure levels of structural knowledge. ARCS scores were measured based on free-recall responses. When two words from the same category were recalled together, the two words were evaluated as well structured by the participant. In this case, the "same category" would mean that the participant sorted the two terms into the same group in the sorting task. ARCS scores ranged between the numerical values of 0 and 1 , and a higher score indicates a higher level of structural knowledge.

\subsubsection{Relationship between Assessment Skills and Structural Knowledge}

Participants were divided into two groups based on their post-examination ARCS score in the sixth trial: Participants who scored over 0.90 points were assigned to the high-structured group and participants who scored less than 0.80 points were assigned to the low-structured group. We compared the mean scores of the assessment skills of two groups.

\subsubsection{Data Analysis}

Data analysis was performed using the statistical program ANOVA4 on the Web. The level of significance was set at $p<0.05$. 
Table 3. Checklist to evaluate assessment skills.

\begin{tabular}{|c|c|c|c|}
\hline \multirow{2}{*}{ Items } & \multicolumn{3}{|c|}{ Assessment categories } \\
\hline & $\begin{array}{l}\text { Information included } \\
\text { in the case }\end{array}$ & $\begin{array}{l}\text { Information not included } \\
\text { in the case }\end{array}$ & $\begin{array}{l}\text { Conclusions and } \\
\text { reasons }\end{array}$ \\
\hline \multirow{6}{*}{ Nutrition } & Low TP & Height/weight & \\
\hline & Low ALB & Skin & \\
\hline & Low Hb & Intravenous drip & \\
\hline & Eating half of a meal & Patient's percention of & \\
\hline & Calorie intake is half & nutrition & \\
\hline & $\begin{array}{l}\text { Saying "I might not be hungry } \\
\text { because I do not walk" }\end{array}$ & & \\
\hline \multirow{2}{*}{ Activity level } & $\begin{array}{l}\text { Usually in a dorsal position } \\
\text { on the bed }\end{array}$ & Scar pain & \\
\hline & $\begin{array}{l}\text { Patient can walk around } \\
\text { the ward freely }\end{array}$ & & \\
\hline Constipation & No stool for 3 days & Liquid intake & \\
\hline \multirow{4}{*}{$\begin{array}{l}\text { Problems and factors } \\
\text { of the issues }\end{array}$} & & & Loss of appetite \\
\hline & & & Low activity level \\
\hline & & & Constipation \\
\hline & & & $\begin{array}{l}\text { Imbalanced nutrition: less than } \\
\text { body requirements }\end{array}$ \\
\hline \multirow{2}{*}{ Bedsores } & $\begin{array}{l}\text { Pressure on the same parts } \\
\text { of the body for a long time }\end{array}$ & Bedsores skin around sacrum & He can walk by himself \\
\hline & Low nutrition & & $\begin{array}{l}\text { Low risk of impaired tissue } \\
\text { integrity/progress surveillance }\end{array}$ \\
\hline \multirow{5}{*}{ Infections } & High CRP & Infection & \multirow{2}{*}{$\begin{array}{l}\text { He was treated with lung } \\
\text { lobectomy } 5 \text { days ago }\end{array}$} \\
\hline & Low nutrition & Vital signs & \\
\hline & & Red/flushed & \multirow{3}{*}{ Normal reaction for surgery } \\
\hline & & Scar pain & \\
\hline & & Scar intention & \\
\hline Score & 13 & 13 & 8 \\
\hline
\end{tabular}

\subsection{Ethical Considerations}

The Ethics Committee of Hirosaki University Graduate School of Medicine gave ethical approval for this study. Participants were provided with a complete explanation about the objective and process of the study. Written consent was obtained from each participant. All participants voluntarily participated and were free to withdraw from the study at any time.

\section{Result}

We analyzed the data of the 13 participants who participated in both pre- and post-examinations (before and after clinical practice). The data of 4 participants who participated in only the pre-examination were excluded.

\subsection{Structural Knowledge}

ARCS scores as indices for levels of structural knowledge were calculated for the six trials of each participant. 
Mean ARCS scores of trials 1 to 6 and mean ARCS scores of pre- and post-examinations were compared (Figure 1).

As a result of analysis of variance, mean ARCS scores increased with each trial in both pre- and post-examinations. The score of the sixth trial became the peak value $(F[5,60]=102.54, p<0.001)$. Also, mean ARCS scores were significantly higher post-examination compared with pre-examination $(F[1,12]=11.14, p<0.01)$. There were no interactions between the trials and pre- and post-examinations $(F[5,60]=0.363, p>0.10)$.

\subsection{Nursing-Scene Task}

Each participant's assessment protocol was derived from the nursing-scene task responses. We evaluated each participant's assessment skills using the checklist and calculated the average score of each assessment category for each participant. We then compared the mean average scores of the pre- and post-examinations (Figure 2).

The main effect of the pre- and post-examinations was significant based on variance analysis $(F[1,12]=$ 27.64, $p<0.001$ ). Assessment skill scores of the post-examination were higher than those of the pre-examination. Also, the main effect of the category was significant $(F[2,24]=42.48, p<0.001)$. Multiple comparisons by Ryan's method were conducted. As a result, there were significant differences between "information included in the case" and "information not included in the case" $(t[24]=9.17)$, between "information included in the case" and "conclusions and reasons" $(t[24]=3.77)$, and between "information not included in the case" and "conclusions and reasons" $(t[24]=5.40)$. The average score of "information included in the case" was the highest and the average score of "information not included in the case" was the lowest. There were no interactions between the assessment categories and pre- and post-examinations $(F[2,24]=1.71, p>0.10)$.

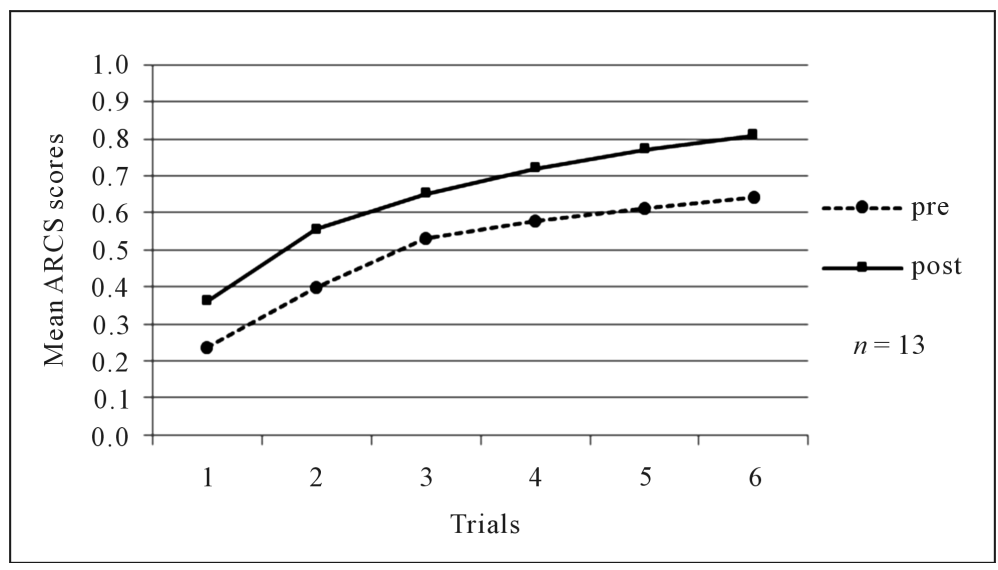

Figure 1. Mean ARCS scores of each trial.

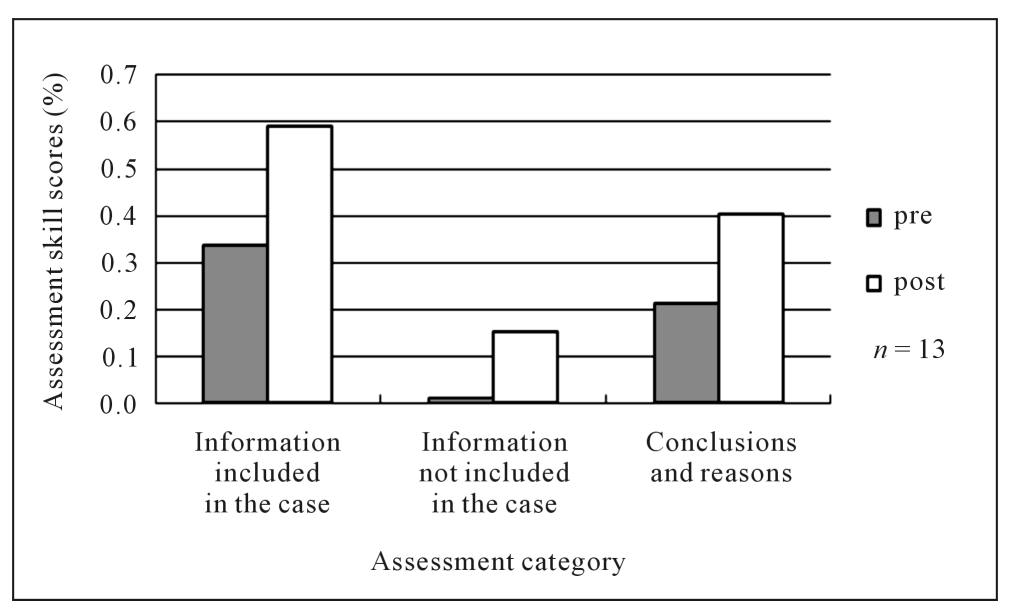

Figure 2. Mean assessment skill scores of pre- and post-examinations. 


\subsection{Relationship between Assessment Skills and Levels of Structural Knowledge}

Participants were divided into two groups based on their post-examination ARCS scores in the sixth trial. Five participants who scored over 0.90 points were assigned to the high-structured group, and 5 participants who scored less than 0.80 points were assigned to the low-structured group. Participants who scored $0.81-0.89$ points were excluded from this analysis.

Mean average scores of each category were compared between the low-structured group and high-structured group (Figure 3). The main effect of the groups was significant based on variance analysis $(F[1,8]=10.57, p<$ $0.05)$. The main effect of the assessment category was also significant $(F[2,16]=47.59, p<0.001)$. Furthermore, a significant interaction was found between levels of structural knowledge and assessment category $(F[2,16]=5.80, p<0.05)$. Simple main effects were tested for structural knowledge and assessment categories. As a result, the simple main effect of structural knowledge in "conclusions and reasons" was significant $(F[1,24]$ $=21.15, p<0.001$ ). Scores of the high-structured group were significantly higher in "conclusions and reasons" compared with those of the low-structured group. Also, the simple main effect of structural knowledge in "information not included in the case" was marginally significant $(F[1,24]=3.31, p<0.10)$. Scores in the high-structured group tended to be higher than those in the low-structured group. The simple main effect of structural knowledge in "information included in the case" was not significant.

\section{Discussion}

\subsection{Memory Model and Structural Knowledge in Cognitive Psychology}

First, an overview of the memory model and structural knowledge in cognitive psychology will be followed by a discussion of the structural knowledge of the nursing students. In cognitive psychology, a memory model is considered an operation of acquiring new information. According to Baddeley [10], in a memory model, new information is operated on in working memory to become long-term memory. New information is associated with long-term memory knowledge through the working memory operation. In this study, acquiring knowledge as long-term memory is called "memorizing." When memorizing new information, it is associated with already existing knowledge of situations, with the information used to facilitate structural knowledge and its activation when needed [10]. In nursing science, when acquiring new information, associating new information with a clinical setting related to the information facilitates activation of structural knowledge during assessment, which makes possible the effective utilization of knowledge.

\subsection{Levels of Structural Knowledge and Assessment Skills}

Next, we will discuss the relationship between levels of structural knowledge and assessment skills. Assessment is the collection and interpretation of information. Its purpose is to decide whether or not a problem exists, such as a problem requiring nursing intervention or a potential problem [2]. Therefore, we analyzed the categories

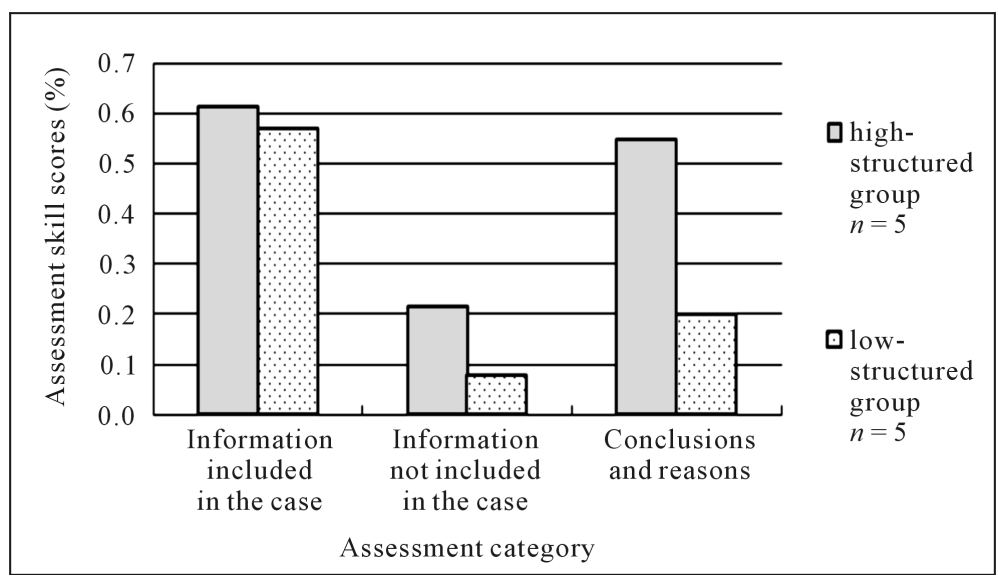

Figure 3. Relationship between assessment skills and levels of structural knowledge. 
"information paid attention to" and "conclusions and reasons" based on responses to a nursing-scene task. In "information paid attention to", we found no difference between students with low and high levels of structural knowledge in the percentage who mentioned information that was directly provided in the task case. The high-structured group tended to mention necessary information that was related to but not directly provided in the task case. The percentage who mentioned a decision made along with its reasons was also significantly higher in the high-structured group.

Benner [11] explains five steps to nursing mastery. Because novices lack experience to base their decisions on, phenomena must be understood based on objective data from an observable patient. Therefore, novices barely understand a given situation. In the next step, advanced beginner-level nurses have knowledge and experience to base their decisions on. Therefore, for patients presenting similar symptoms as a previous patient, nurses are able to determine what to observe and what type of nursing is needed [11]. The low-structured group in this study corresponds to Benner's novice level. Although nurses in the low-structured group were able to pay attention to information directly mentioned in the task case as well as those in the high-structured group, they were unable to mention any related information. They were unable to use unmentioned knowledge because their knowledge of nutritional-metabolic patterns was not structured. In contrast, knowledge of nutritional-metabolic patterns was structured in the high-structured group, making it possible for them to acquire and activate highly related knowledge about the case. Benner [11] also stated that novices have difficulty comprehensively analyzing data due to a fragmentary understanding of a situation. Only a low percentage of the low-structured group was able to state their decisions in relation to their reasons, which is also a characteristic of Benner's novice level. Although the low-structured group was able to notice important information, they could make a decision using one piece of information but had difficulty making a decision that demanded comprehensive understanding of several pieces of information because of their fragmentary knowledge of the situation.

These above-mentioned assessment characteristics will be discussed from the perspective of cognitive psychology. In one study, Gobband Chi [12] analyzed the difference between an expert and a novice from the same field that was shown a picture related to their field and asked to comment about it. First, there were no differences in visually observed characteristics, although experts commented more often about characteristics not visually observed. Second, whereas novices only listed the characteristics, experts talked about details related to a characteristic they had commented about. In a study comparing the ways in which experts and novices solved a physics problem, Chi [6] found that experts predicted unwritten events based on information given to solve the problem. Chi [6] stated that information gained in fragments might not be appropriately used and must be structured around a basic concept for it to be appropriately used. In the present study, the assessment characteristics of the high-structured group match that of Chi's characteristics of an expert. Because information was structured and the relationships between the information were understood, decisions were not made using one piece of information but rather by over viewing the information comprehensively to determine whether the problem required nursing intervention.

\subsection{Structural Knowledge before and after Clinical Practice}

Here, we discuss the mechanism of structural knowledge after clinical practice according to characteristics of the students' structural knowledge before and after clinical practice. In this study, the nurses' knowledge was well-structured after their clinical practice. During clinical practice, students collected information from primary patients, assessed the type of nursing needed, and actually provided the needed nursing. When learning new information, being conscious of how the information will be used in actual nursing serves to facilitate structuring of knowledge. Murayama [13] showed that test format affects the choice of learning strategy such that the group that predicted a written test chose strategies that emphasized understanding meaning or flow of events compared with the group that predicted a cloze test. Murayama's findings suggest that the purpose of learning affects learning strategies and structural knowledge. Although students acquire knowledge about disorders and nursing through lectures before their clinical practice, their consciousness of post-lecture testing results in their choice of learning strategies that are most advantageous for the test. Therefore, structural knowledge will be based on each lecture. Information given in one lecture will be structured together whereas information from other lectures and the condition of patients in a clinical setting might not be linked together. In contrast, knowledge needed in a clinical setting can be specifically imagined through clinical practice. Students can further their studies by being aware that the knowledge will be used in a clinical setting. In a clinical setting, one patient might show several 
symptoms that require comprehensive consideration of the situation for nursing. Therefore, through clinical practice, one must integrate fragmentary information by comprehensive assessment of the situation. Thus lecture-based knowledge facilitates the connection between obtained knowledge and knowledge from clinical settings.

Experience in clinical practice improves students' structural knowledge for its effective utilization in making assessments in a clinical setting. Knowledge required to make assessments is utilized to facilitate structural knowledge, which makes it possible to make assessments through comprehensively understanding the situation. It was clarified that well-structured knowledge facilitates the development of the assessment skills. Therefore, promoting education that facilitates the structuring of knowledge is important for improving students' assessment skills.

\section{Conclusions}

1) Scores of structural knowledge regarding nutrition evaluation were significantly higher after clinical practice.

2) Scores of assessment skills after clinical practice were raised significantly compared with those before clinical practice.

3) Regarding the relationship between levels of structural knowledge and assessment skills, information-collecting scores did not differ between the low- and high-structured groups, although the "conclusions and reasons" score was significantly higher in the high-structured group. The high-structured group tended to mention information that was not stated in the task case.

4) Through clinical practice, students develop structural knowledge that can be utilized in a clinical setting to improve assessment skills.

\section{Limitations}

In this study, we compared assessment skills and levels of structural knowledge using randomly chosen data from students before and after their clinical practice without controlling the content of clinical practice. Therefore, whether the content of clinical training actually promoted structural knowledge remains uncertain. Discussions were based on clinical practice experience and past studies. Future research should investigate teaching strategies for acquiring structural knowledge that can be utilized in a clinical setting, and its effects on assessment skills must be tested. In addition, the number of participants in this study was low because the experiments took a long time and few students volunteered. Consequently, our analyses were based on a small amount of data from a limited sample. Further studies with larger sample sizes and more diverse populations (e.g., other schools, other grades) are needed.

\section{Acknowledgements}

This study was supported by JSPS KAKENHI Grant-in-Aid for Young Scientists (B) Grant Number 25862099. The authors are grateful to the participants of this study, who provided us with invaluable information.

\section{References}

[1] The American Nurses Association (2015) The Nursing Process. http://www.nursingworld.org/EspeciallyForYou/What-is-Nursing/Tools-You-Need/Thenursingprocess.html

[2] Gordon, M. (2008) Assess Notes: Nursing Assessment \& Diagnostic Reasoning. F.A. Davis Company, Philadelphia.

[3] Sasabe, M., Manabe, E., Fujita, J., Komatsu, M., Mitsuhashi, M., Babaguchi, Y., Nishida, N., Nakagawa, M., Fukumoto, M. and Okayama, Y. (2009) Evaluation of Clinical Nursing Skills of University Students in Nursing Course at Time of Graduation. Bulletin of School of Nursing Kyoto Prefectural University of Medicine, 18, 55-63. (In Japanese with English Summary)

[4] Komatsu, M., Izumi, M. and Okubo, Y. (2011) Nursing Practical Skills and Educational Issues among Nurses at Completion of Nursing Curriculum and Nurses in the First to Third Years after Graduation. Journal of Kyoto Prefectural University of Medicine, 120, 781-791. (In Japanese with English Summary)

[5] Yura, H. and Walsh, M.B. (1983) The Nursing Process: Assessing, Planning, Implementing, Evaluating. Prentice-Hall, Inc., Connecticut. 
[6] Chi, M.T.H., Feltovich, P.J. and Glaser, R. (1981) Categorization and Representation of Physics Problems by Experts and Novices. Cognitive Science, 5, 121-152. http://dx.doi.org/10.1207/s15516709cog0502_2

[7] Hemming, T.R. and Jennrich, J.A. (2013) Using Standardized Patients to Strengthen Nurse Practitioner Competency in the Clinical Setting. Nursing Education Perspectives, 34, 118-121. http://dx.doi.org/10.5480/1536-5026-34.2.118

[8] Mitsuki, S., Mouri, T., Sasagawa, H. and Takishita, Y. (2011) The Effectiveness and Areas of Program, Which Is Part of the Fundamental Nursing Education to Improve Clinical Nursing Competence. Journal of Kyoto Prefectural University of Medicine, 120, 769-780. (In Japanese with English Summary)

[9] Inoki, S. (1989) Comparison of Measures of Category Clustering for Children's Memory. Bulletin of the Faculty of Home Economics Hiroshima Woman's University, 25, 45-52. (In Japanese with English Summary)

[10] Baddeley, A.D. (1997) Human Memory: Theory and Practice (Revised Edition). Psychology Press, East Sussex.

[11] Benner, P. (2001) From Novice to Expert. Prentice Hall Health, New Jersey.

[12] Gobb, C. and Chi, M. (1986) How Knowledge Is Structured and Used by Expert and Novice Children. Cognitive Development, 1, 221-237. http://dx.doi.org/10.1016/S0885-2014(86)80002-8

[13] Murayama, K. (2004) Effects of Test Format on Learning Strategy and Perceived Utility. The Japanese Journal of Psychology, 75, 262-268. (In Japanese with English Summary). http://dx.doi.org/10.4992/jjpsy.75.262 